\title{
CZK3, a MAP Kinase Kinase Kinase Homolog in Cercospora zeae-maydis, Regulates Cercosporin Biosynthesis, Fungal Development, and Pathogenesis
}

\author{
Won-Bo Shim and Larry D. Dunkle \\ Crop Production and Pest Control Research, U.S. Department of Agriculture-Agricultural Research Service, Department of \\ Botany and Plant Pathology, Purdue University, West Lafayette, IN 47907-2054 U.S.A.
}

Submitted 3 March 2003. Accepted 30 May 2003.

\begin{abstract}
The fungus Cercospora zeae-maydis causes gray leaf spot of maize and produces cercosporin, a photosensitizing perylenequinone with toxic activity against a broad spectrum of organisms. However, little is known about the biosynthetic pathway or factors that regulate cercosporin production. Analysis of a cDNA subtraction library comprised of genes that are up-regulated during cercosporin synthesis revealed a sequence highly similar to mitogen-activated protein (MAP) kinases in other fungi. Sequencing and conceptual translation of the full-length genomic sequence indicated that the gene, which we designated $C Z K 3$, contains a 4,119-bp open reading frame devoid of introns and encodes a 1,373-amino acid sequence that is highly similar to Wis4, a MAP kinase kinase kinase in Schizosaccharomyces pombe. Targeted disruption of $C Z K 3$ suppressed expression of genes predicted to participate in cercosporin biosynthesis and abolished cercosporin production. The disrupted mutants grew faster on agar media than the wild type but were deficient in conidiation and elicited only small chlorotic spots on inoculated maize leaves compared with rectangular necrotic lesions incited by the wild type. Complementation of disruptants with the $C Z K 3$ open reading frame and flanking sequences restored wild-type levels of conidiation, growth rate, and virulence as well as the ability to produce cercosporin. The results suggest that cercosporin is a virulence factor in $C$. zeae-maydis during maize pathogenesis, but the pleiotropic effects of $C Z K 3$ disruption precluded definitive conclusions.
\end{abstract}

Additional keywords: pathogenicity, signaling.

Corresponding author: L. D. Dunkle; E-mail: dunkle@purdue.edu; Telephone: +1-765-494-6076.

Current address of W.-B. Shim: Department of Plant Pathology and Microbiology, Texas A\&M University, College Station, TX 77843-2132.

Nucleotide sequence data for CZK3 is available in the GenBank database under accession number AY242849.

Names are necessary to report factually on available data. However, the USDA neither guarantees nor warrants the standard of the product, and the use of the names implies no approval of the product to the exclusion of others that also may be suitable.

This article is in the public domain and not copyrightable. It may be freely reprinted with customary crediting of the source. The American Phytopathological Society, 2003.
Many plant pathogenic fungi produce low molecular weight secondary metabolites that have a significant impact on the outcome of the host-pathogen interaction (Wolpert et al. 2002; Yoder 1980). Like many species of Cercospora (Assante et al. 1977; Fajola 1978; Lynch and Geoghegan 1979), C. zeae-maydis, the causal agent of gray leaf spot of maize, produces cercosporin (Gwinn et al. 1987; Jenns et al. 1989; Wang et al. 1998), a photosensitizing secondary metabolite that is toxic to a diverse array of organisms (Daub and Ehrenshaft 2000). Lightactivated cercosporin reacts with oxygen to generate singlet oxygen and superoxide, which induce lipid peroxidation and damage the cell membrane, resulting in leakage of cytoplasmic contents and, eventually, cell death (Daub 1982; Daub and Ehrenshaft 2000). Several observations support a role of cercosporin in pathogenesis. In many diseases caused by fungi that produce cercosporin or other structurally related phytotoxic perylenequinones, disease severity is correlated with light exposure in accord with the mode of action of these compounds (Daub and Ehrenshaft 2000). Also, cercosporin has been isolated from tissues infected with Cercospora spp. (Fajola 1978; Kuyama and Tamura 1957; Velicheti and Sinclair 1994; Venkataramani 1967), although such observations alone do not causally link cercosporin production with pathogenesis.

The most compelling evidence for the involvement of cercosporin in pathogenesis was provided by Upchurch and associates in their work on the soybean pathogen, C. kikuchii. UVinduced mutants selected for defects in cercosporin production on agar media were found to be unable to incite typical necrotic lesions on soybean leaves and could not be isolated from small chlorotic spots (Upchurch et al. 1991). Subsequent studies focused on $C F P$, a gene encoding a membrane transport protein required for secretion of cercosporin, and showed that disruption of $C F P$ resulted in a substantial reduction in cercosporin synthesis and greatly reduced virulence on soybean leaves (Callahan et al. 1999). Collectively, those results suggest that cercosporin is required for wild-type virulence in C. kikuchii. Despite the significance of cercosporin in hostpathogen interactions, the biosynthetic pathway of cercosporin is unknown, and the pathological consequences of its action in gray leaf spot development have not been characterized.

Toward the goal of identifying genes involved in the biosynthesis of cercosporin and ultimately defining its role in gray leaf spot, we constructed a cDNA subtraction library of genes that are up-regulated during cercosporin production in $C$. zeaemaydis (Shim and Dunkle 2002). We grouped cDNA fragments with high similarity to genes in the GenBank database into 
nine categories based on predicted functions of the encoded polypeptides (Shim and Dunkle 2002). Genes in the signal transduction category were of particular interest. Protein kinases of filamentous fungi, e.g., mitogen-activated protein (MAP) kinases, protein kinase $\mathrm{C}$, and cyclic AMP-dependent protein kinases have been shown to play critical roles in the regulation of fungal development and pathogenesis (Dickman and Yarden 1999). The MAP kinase pathway, one of the most extensively studied signal transduction pathways in plant pathogenic fungi (Xu 2000), controls several developmental stages essential for pathogenicity of the rice blast fungus Magnaporthe grisea (Tenjo et al. 1999; Xu and Hamer 1996; Xu et al. 1998) and the corn smut pathogen Ustilago maydis (Banuette and Herskowitz 1994; Mayorga and Gold 1999; Muller et al. 1999). However, the role of signal transduction pathways that regulate and integrate fungal secondary metabolism associated with development and plant pathogenesis has not been thoroughly documented. A limited number of reports have investigated the involvement of signal transduction pathways in regulating secondary metabolism and fungal differentiation. For example, sterigmatocystin biosynthesis and conidiation in Aspergillus nidulans are linked through a common requirement for inactivation of a heterotrimeric $G$ protein-dependent signaling pathway that stimulates growth while repressing both conidiation and sterigmatocystin biosynthesis (Adams and Yu 1998; Hicks et al. 1997; Yu et al. 1996). Similarly, disruption of a C-type cyclin in Fusarium verticillioides, the causal agent of maize ear rot, blocked both fumonisin biosynthesis and conidiation when the fungus was grown on maize kernels (Shim and Woloshuk 2001). However, the paucity of genetic information in plant pathogenic fungi com-

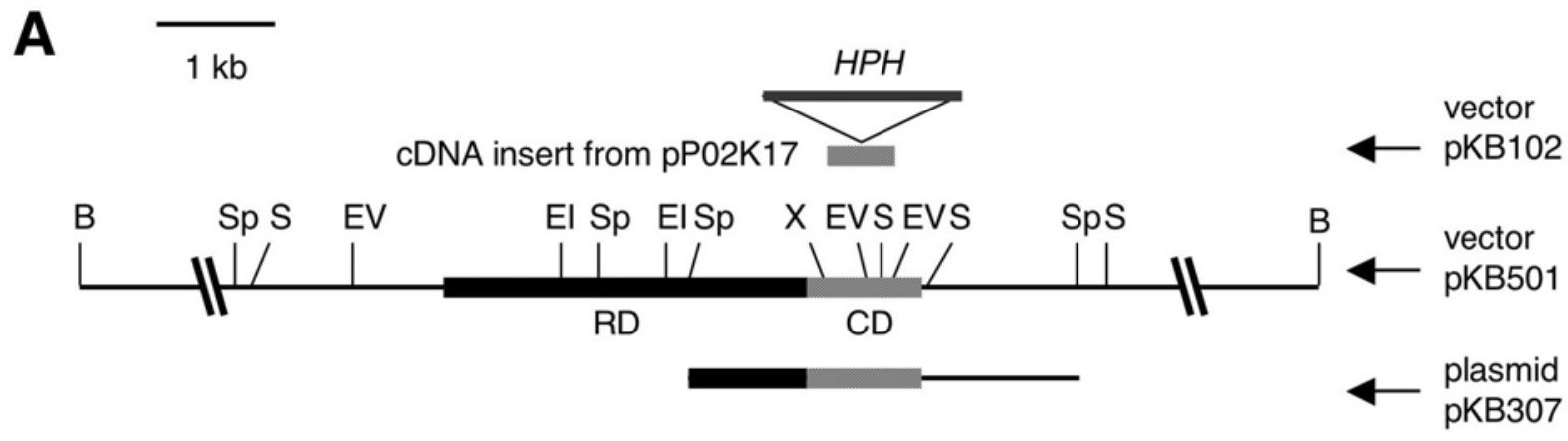

B Czk3
FgMAPKKK
NcMAPKKK
MgSsk2
Wis4

Czk3
FgMAPKKK
NcMAPKKK
MgSsk2
Wis4

Czk3
FgMAPKKK
NcMAPKKK
MgSsk2
Wis4

Czk3
FgMAPKKK
NcMAPKKK
MgSsk2
Wis4
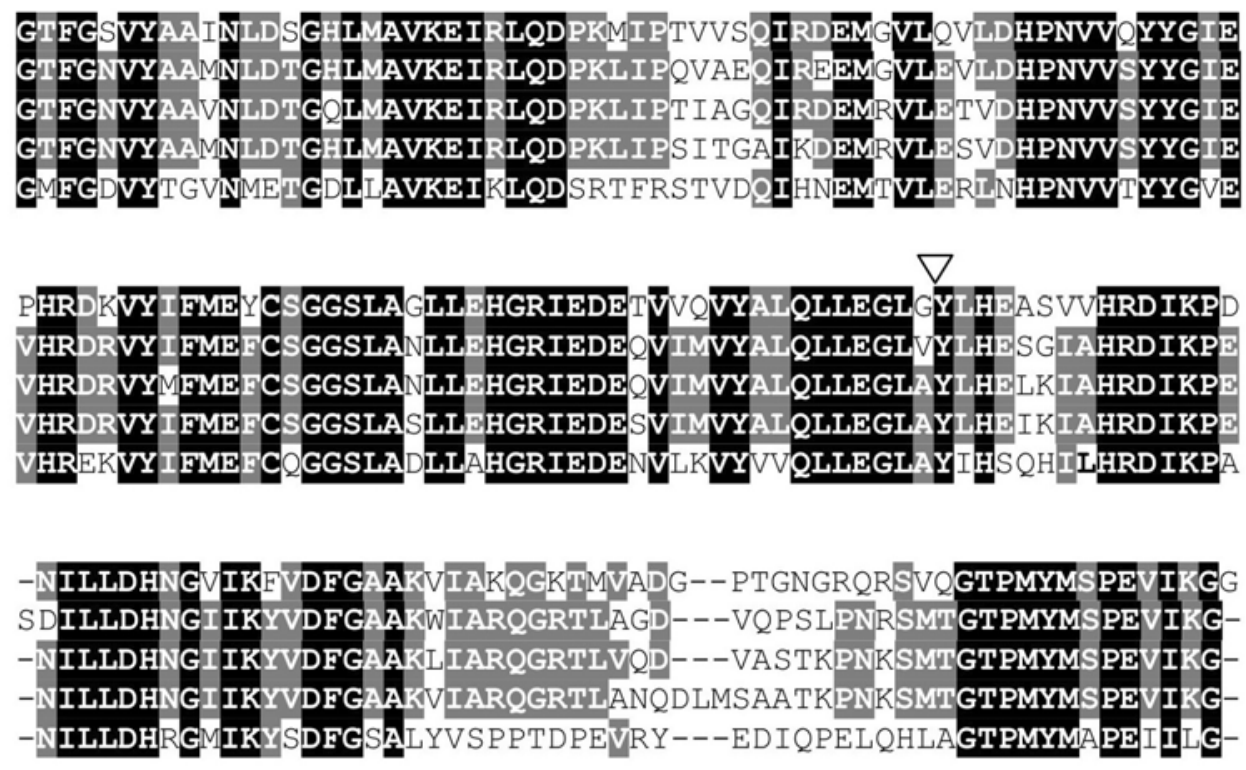

Fig. 1. The CZK3 gene in Cercospora zeae-maydis. A, Restriction map of the genomic region containing CZK3, including the catalytic domain (CD) and regulatory domain $(\mathrm{RD})$. The disruption vector pKB102 was generated by inserting the 1.4-kb hygromycin-resistance cassette $(H P H)$ into the $\mathrm{cDNA}$ insert of clone cP02K17 from the subtraction library. A 3-kb genomic DNA fragment (pKB307) was subcloned for sequencing. The 11-kb BamHI fragment from cosmid bcM3K was cloned into the pBARGPE1 vector to generate vector pKB501 for complementation. Restriction sites are: B = BamHI, EI = EcoRI, $\mathrm{EV}=$ EcoRV, $\mathrm{S}=$ SalI, $\mathrm{Sp}=S p h \mathrm{I}$, and $\mathrm{X}=$ XbaI. B, Amino acid alignment of catalytic domains of Czk3, Fusarium graminearum MAPKKK, Neurospora crassa MAPKKK, MgSsk2 (MAPKKK homolog in Magnaporthe grisea), and Wis4 (MAPKKK homolog in Schizosaccharomyces pombe) via CLUSTAL $\mathrm{W}$. Amino acids that are common to all five proteins are indicated by white letters on a black background, and residues present in three or four of the five proteins are indicated by a gray background. Gaps introduced for alignment are indicated by dashes. The open triangle indicates the site of insertion of $H P H$ in the disruption vector. 
plicates investigations of the signal transduction pathways that regulate and integrate fungal differentiation, secondary metabolism, and plant pathogenesis.

In this study, we investigated the involvement of a signal transduction gene in cercosporin biosynthesis by $C$. zeae-maydis. We describe $C Z K 3$, a gene that is highly similar to the MAP kinase kinase kinase (MAPKKK) WIS4, which encodes a component of a signal transduction pathway in Schizosaccharomyces pombe that is activated by heat shock, oxidative stress, and limited nutrition (Samejima et al. 1997). Results of targeted gene disruption and complementation of disrupted mutants indicate that $C Z K 3$ performs a critical regulatory function in fungal development and pathogenesis as well as cercosporin biosynthesis in C. zeae-maydis.

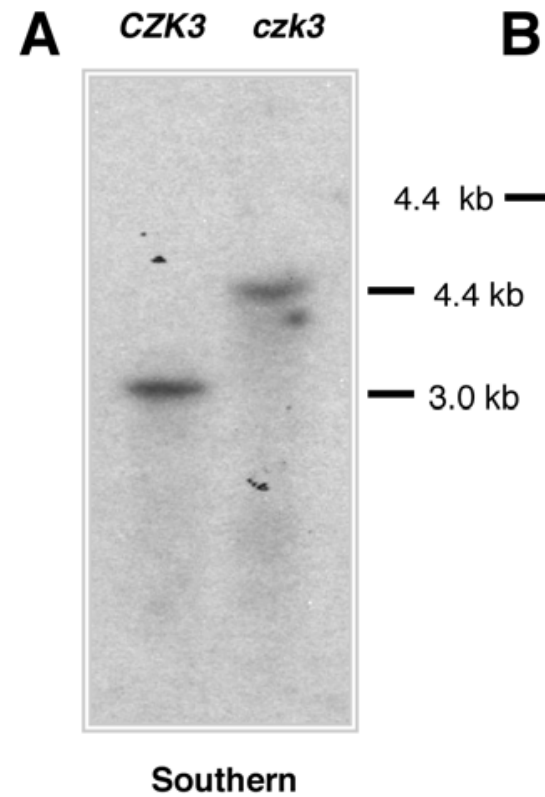

B CZK3

czk3

Fig. 2. Southern and Northern blot analyses of $C Z K 3$ in Cercospora zeae-maydis wild type $(C Z K 3)$ and gene disruption mutant $(c z k 3)$. A, Genomic DNA $(10 \mu \mathrm{g})$ from wild-type and mutant isolates were digested with $S p h$ I and subjected to electrophoresis in a $1.0 \%$ agarose gel. B, Total RNA samples $(15 \mu \mathrm{g})$ from wild-type and mutant mycelia grown in $0.2 \times$ potato dextrose broth were subjected to electrophoresis in a $1.2 \%$ denaturing agarose gel. The gel was stained with ethidium bromide to confirm uniformity of loading (rRNA). The ${ }^{32} \mathrm{P}$-labeled $C Z K 3 \mathrm{cDNA}$ insert ( $380 \mathrm{bp}$ ) from cP02K17 was used as a probe for both Southern and Northern blot analyses.

\section{RESULTS}

Identification and characterization of the $C Z K 3$ gene encoding a putative MAPKKK.

From the signal transduction category of a C. zeae-maydis cDNA subtraction library (Shim and Dunkle 2002), we identified a clone (cP02K17) containing a 380-bp cDNA insert (Fig. 1A) that is highly similar (69\% amino acid identity) to the serine/threonine kinase domain of Ssk2, a MAPKKK in the conserved HOG pathway of Saccharomyces cerevisiae (Banuett 1998). We have designated the gene represented by this cDNA as $C Z K 3$ (to indicate a MAPKKK from $C$. zeae-maydis). The cDNA fragment was used as the probe to identify a cosmid clone (bcM3K) that contained this gene from the C. zeae-maydis genomic library. To obtain the genomic sequence of $C Z K 3$, we subcloned and sequenced a 3-kb genomic DNA fragment from bcM3K (pKB307) (Fig. 1A). As a more efficient and supplemental approach to subcloning and plasmid walking, we generated random transposon insertions in bcM3K and used the primer sites on the transposon for sequencing (discussed below). Over $90 \%$ of the cosmid sequence was generated by this method, and the sequence fragments were assembled into a contig containing the entire open reading frame of $C Z K 3$ and $5^{\prime}$ and $3^{\prime}$ flanking sequences (accession number AY242849).

Sequencing results revealed a 4,119-bp open reading frame devoid of introns. Conceptual translation resulted in a 1,373-amino acid polypeptide (Fig. 1) that is highly similar to previously characterized serine and threonine protein kinases, which have a highly conserved catalytic domain in the C-terminal portion and a more variable $\mathrm{N}$-terminal region that is suggested to serve as the regulatory domain (Posas and Saito 1998). BLASTP analysis of the catalytic domain indicated that $\mathrm{Czk} 3$ was most similar to Ssk2 (55\% amino acid identity). However, the full-length deduced amino acid sequence had higher similarity (36\% identity, $56 \%$ similarity) to $S$. pombe Wis4, a MAPKKK in the Spc1 signaling pathway involved in various stress responses (Samejima et al. 1997, 1998). A TBLASTN search against recently completed fungal genome sequences available from the Center for Genome Research, Whitehead Institute (Cambridge, MA, U.S.A.) revealed MAPKKK sequences closely related to Czk3 in Fusarium graminearum (overall $48 \%$ identity, 63\% similarity) and Neurospora crassa (overall $47 \%$ identity, $63 \%$ similarity). Czk3 also is highly similar (overall $48 \%$ identity, $64 \%$ similarity) to a putative MAPKKK in M. grisea that is a homolog of Ssk2 (J.-R. Xu, personal communication). Sequence comparisons of the catalytic domains of the deduced proteins from the above fungi are shown in Figure 1B.

Comparative promoter analysis by TRES (Transcription Regulation Element Search) to search for common elements

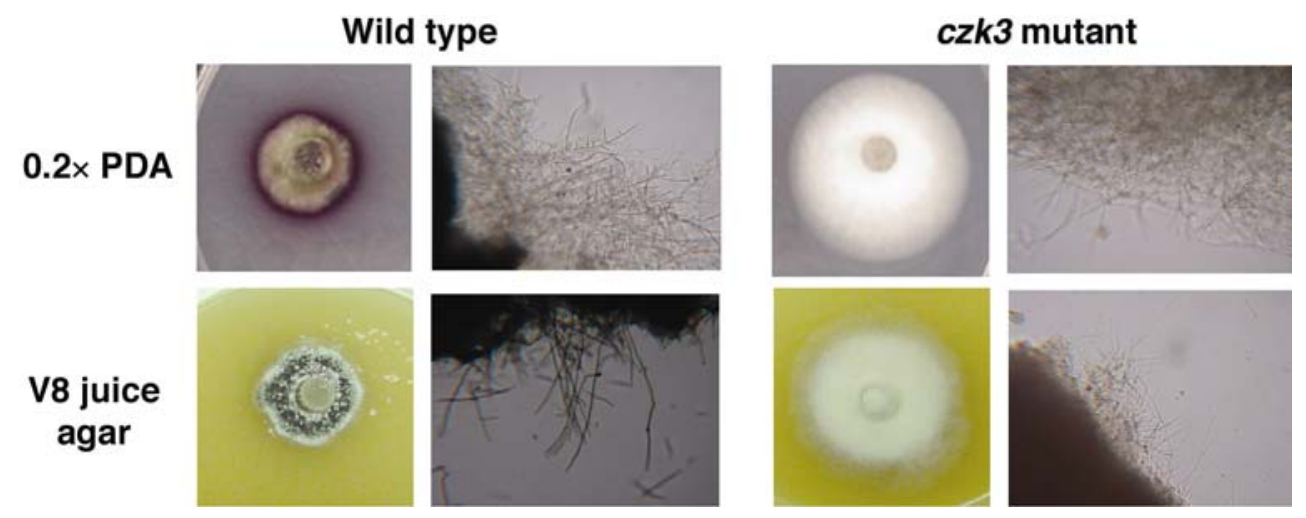

Fig. 3. Colony morphology of the wild type (CZK3) and gene disruption mutant (czk3) of Cercospora zeae-maydis grown on $0.2 \times$ potato dextrose agar (PDA) and V8 juice agar. Fungal isolates were point-inoculated and incubated for one week at $25^{\circ} \mathrm{C}$ under a 14 -h light and 10 -h dark cycle. Isolates grown on $0.2 \times$ PDA and V8 juice agar were examined for cercosporin production and conidiation, respectively. 
in promoter regions of $C Z K 3$ and the related gene in $M$. grise $a$ revealed a cAMP-responsive element ( $85 \mathrm{bp})$, a CAAT box (108 bp), a PhoA-like upstream stimulating factor (307 $\mathrm{bp}$ ), and a GATA-binding factor (411 bp) in the promoter region of $C Z K 3$, all within $1 \mathrm{~kb}$ upstream of the initiation codon. Identification of these cis-acting elements may provide information about the regulation of $C Z K 3$ expression during fungal growth and cercosporin biosynthesis.

\section{Disruption of $C Z K 3$.}

We performed targeted disruption of $C Z K 3$ via homologous recombination to investigate the function of this gene in cercosporin production and pathogenesis by $C$. zeae-maydis. The gene disruption vector $\mathrm{pKB} 102$ was constructed by inserting a hygromycin phosphotransferase $(H P H)$ gene into the 380-bp insert of pP02K17 (Fig. 1A). Hygromycin-resistant transformants were subjected to Southern analysis and were tested for cercosporin production on $0.2 \times$ potato dextrose agar (PDA) (Shim and Dunkle 2002). Southern analysis of genomic DNA from the wild type and transformant M1228-21, which did not produce detectable levels of cercosporin during a period of 14 days, showed that a $3-\mathrm{kb}$ band in the wild type was increased by $1.4 \mathrm{~kb}$ in the mutant, confirming that the $H G H$ gene $(1.4 \mathrm{~kb})$ was inserted into the targeted site in $C Z K 3$ by homologous recombination (Fig. 2).

\section{CZK3 czk3}

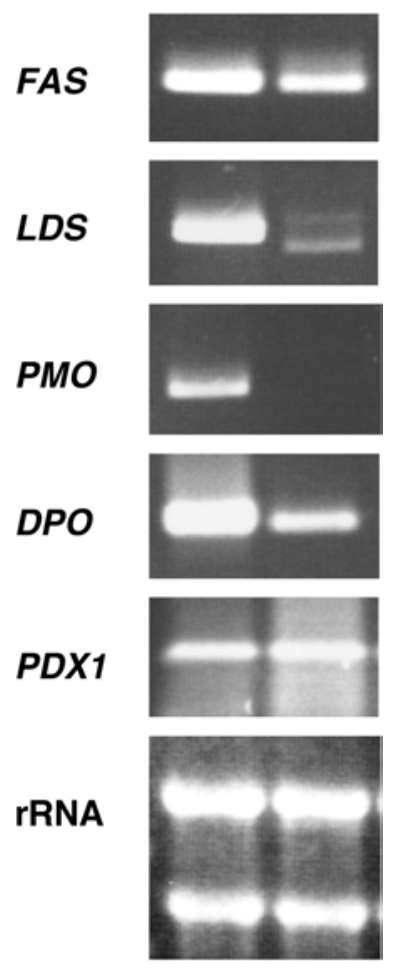

Fig. 4. Effect of $C Z K 3$ disruption on expression of putative cercosporin biosynthesis genes in Cercospora zeae-maydis. Total RNA samples (3 $\mu \mathrm{g})$ from wild-type $(C Z K 3)$ and disruption mutant $(c z k 3)$ mycelia grown in $0.2 \times$ potato dextrose broth were used as templates for reverse transcription-polymerase chain reaction (RT-PCR). Primers specific for fatty acid synthase $(F A S)$, linoleate diol synthase (LDS), P450 monooxygenase $(P M O)$, diphenol oxidase $(D P O)$, and pyridoxine biosynthesis $(P D X 1)$ were used to amplify the respective genes from the two RNA templates. The RT-PCR products $(10 \mu \mathrm{l})$ were subjected to electrophoresis in a $1.0 \%$ agarose gel and were stained with ethidium bromide. The bottom panel (rRNA) shows a 1.2\% denaturing agarose gel stained with ethidium bromide to confirm uniformity of RNA concentrations in the RT-PCR reactions.
To determine whether this gene disruption abolished $C Z K 3$ expression, we performed Northern analysis of total RNA obtained from the wild type and the mutant grown on $0.2 \times$ potato dextrose broth (PDB) for 7 days (Shim and Dunkle 2002). The blot was probed with the 380-bp insert as above. A 4.4-kb transcript present in the wild type was not detected in the disrupted mutant (Fig. 2).

\section{Disruption of $C Z K 3$}

affects fungal development and gene expression.

In addition to abolishing cercosporin production, disruption of CZK3 affected the growth and development of C. zeae-maydis (Fig. 3). The mutant (M1228-21) grew 2.0-, 2.1-, and 1.7-fold faster than the wild type on PDA, V-8 juice agar, and oatmeal agar, respectively. Significantly, the mutant did not produce conidiophores or conidia on V-8 juice agar (Fig. 3), suggesting

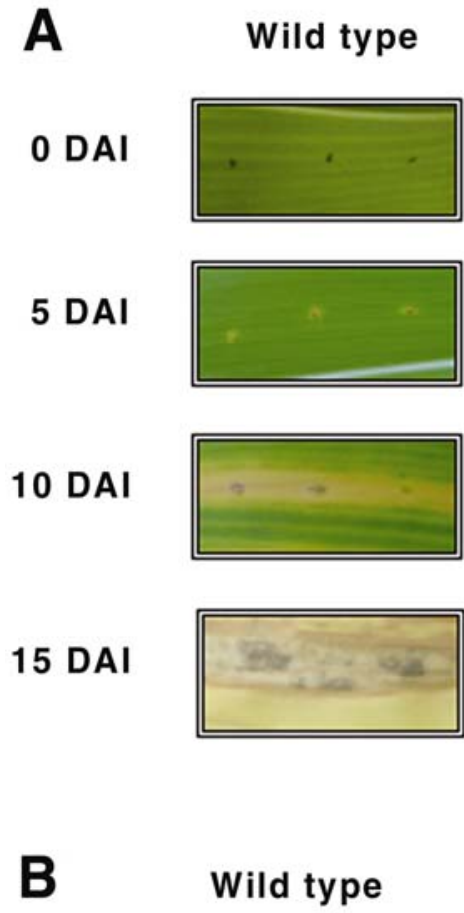

\section{czk3 mutant}
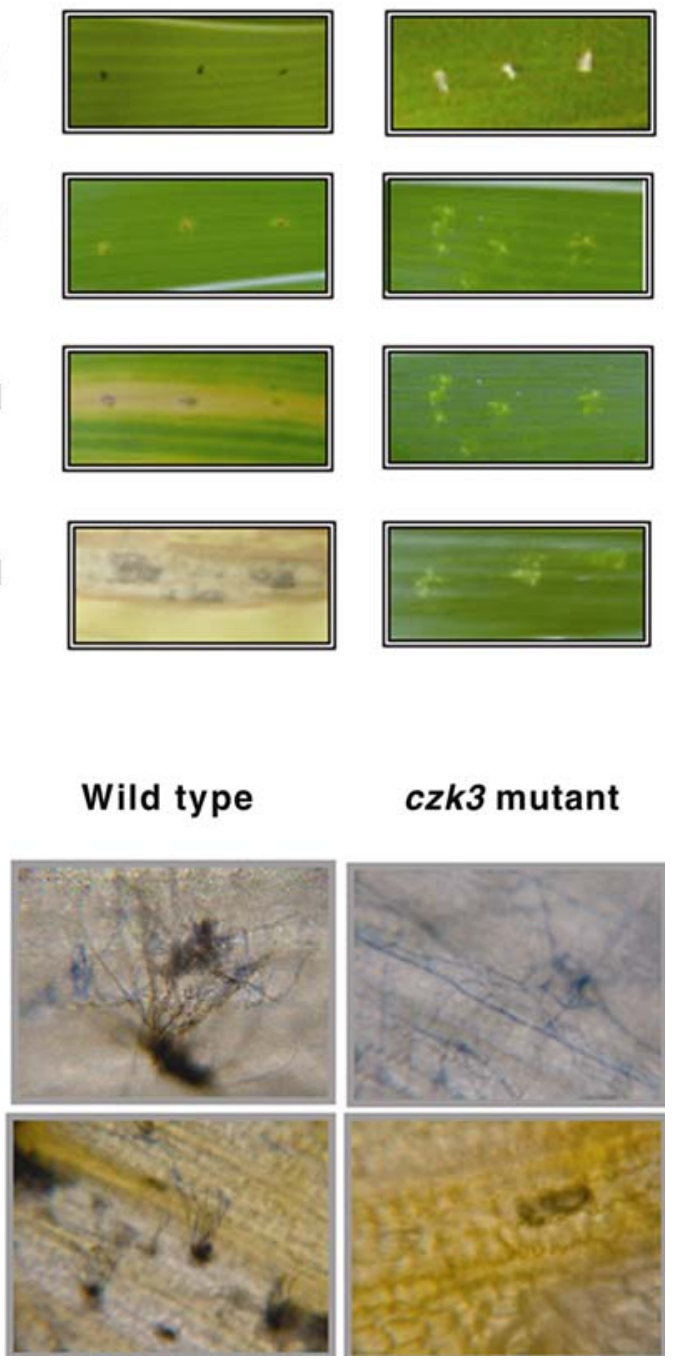

Fig. 5. Requirement of $C Z K 3$ for lesion expansion and disease development in Cercospora zeae-maydis. A, Wild type $(C Z K 3)$ and the gene disruption mutant ( $c z k 3)$ were grown in $0.2 \times$ potato dextrose broth for 7 days at $25^{\circ} \mathrm{C}$ to obtain inoculum. Mycelial blocks $\left(4 \mathrm{~mm}^{2}\right)$ were placed on the abaxial surface of maize leaves (L8 stage). Plants were incubated at $25^{\circ} \mathrm{C}$ in high relative humidity $(>98 \%)$, and disease development was documented every five days after inoculation (DAI). B, Leaf samples bearing lesions (15-DAI samples) were cut into $1-\mathrm{cm}^{2}$ sections and were stained with cotton blue to observe mycelial growth in planta and production of conidiophores and stromata by the wild type and mutant. 
that $\mathrm{Czk} 3$ is critical for asexual reproduction in C. zeae-maydis. In addition, the acute and frequent branching of hyphae observed at the colony margin of the wild type grown on $0.2 \times$ PDA was replaced by sparsely branching hyphae in the mutant.

We investigated the consequence of $C Z K 3$ disruption on the expression of genes with putative functions in cercosporin production (Shim and Dunkle 2002). Expression levels of C. zeaemaydis fatty acid synthase $(F A S)$, linoleate diol synthase $(L D S)$, cytochrome $\mathrm{P} 450$ monooxygenase (PMO), and diphenol oxidase (DPO) genes (Shim and Dunkle 2002), as well as a gene involved in pyridoxine biosynthesis $(P D X 1)$ (Ehrenshaft et al. 1998, 1999) were determined by reverse transcription-polymerase chain reaction (RT-PCR). Total RNA samples from the wild type and the disrupted mutant were used as templates for amplification. Results showed that disruption of $C Z K 3$ substantially decreased expression of $L D S$ and $P M O$, slightly reduced expression of $D P O$, but did not affect expression of $F A S$ or the constitutively expressed $P D X 1$ (Fig. 4). These data suggest that $L D S, P M O$, and $D P O$ are regulated by $C Z K 3$ or the Czk3 protein in the cercosporin biosynthetic pathway but that expression of $F A S$ is independent of $C Z K 3$. Further characterization of these

\section{CZK3 cZk3 CZK3-1}

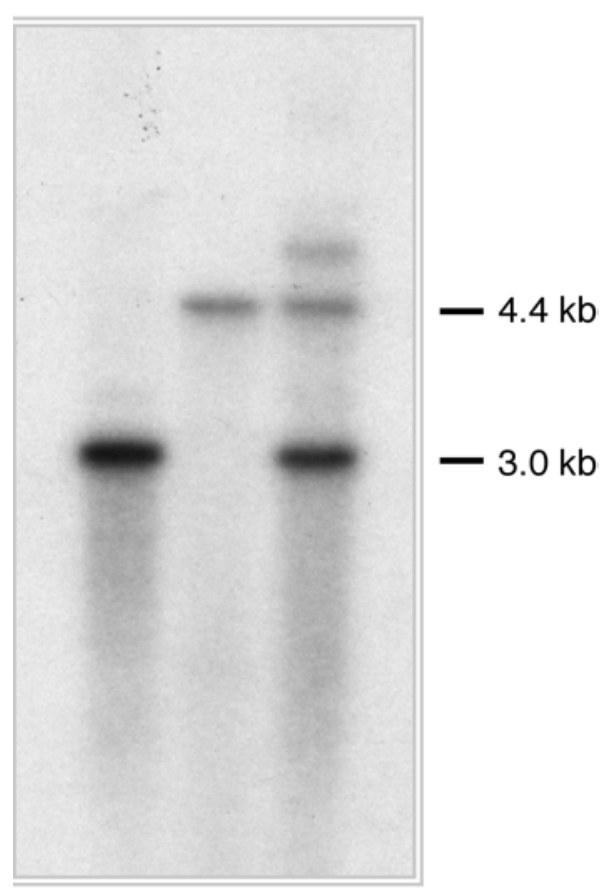

Fig. 6. Southern analyses of CZK3 in Cercospora zeae-maydis. Lane 1, wild type (CZK3); lane 2, gene disruption mutant ( $c z k 3)$; and lane 3, the disrupted mutant complemented with vector pKB501 (CZK3-1). Genomic DNA $(10 \mu \mathrm{g})$ from the wild-type and mutant isolates were digested with $S p h \mathrm{I}$ and were subjected to electrophoresis in a $1.0 \%$ agarose gel. Blots were hybridized with ${ }^{32} \mathrm{P}$-labeled $C Z K 3$ cDNA insert (380 bp) from pP02K17. putative cercosporin biosynthesis genes is necessary for comprehensive understanding of the role of $\mathrm{Czk} 3$ in this pathway.

\section{Disruption of $C Z K 3$ alters pathogenesis.}

We inoculated maize leaves with mycelium of $C$. zeae-maydis wild type (SCOH1-5) and the $c z k 3$ mutant (M1228-21) to test the effect of $C Z K 3$ disruption on pathogenesis (Fig. 5A). The inoculated plants were incubated in the greenhouse in high relative humidity, and symptom development was observed daily for 15 days. Plants inoculated with the mutant developed chlorotic symptoms earlier than those inoculated with the wild type, but symptoms induced by the mutant failed to progress further. Leaves inoculated with the wild type developed chlorotic symptoms that were less severe than those elicited by the $c z k 3$ mutant by 5 days after inoculation (DAI). However, by $10 \mathrm{DAI}$, the severity of chlorosis induced by the wild type had markedly exceeded that caused by the disrupted mutant and, by 15 DAI, rectangular necrotic lesions characteristic of gray leaf spot symptoms had developed only on the leaves inoculated with the wild type (Fig. 5A).

We excised the lesions (15 DAI) and surrounding area from leaves inoculated with the wild type and the mutant and examined them for fungal growth and disease development (Fig. $5 B)$. The wild-type isolate grew more vigorously and colonized maize leaf tissue more extensively than the mutant. The most striking difference was the absence of stromata and conidiophore development in the mutant. Thus, development of these reproductive structures in the mutant was completely blocked in maize leaves as well as on agar media, indicating that the defect in $c z k 3$ in vitro is not relieved in planta. Despite the sparse growth and absence of reproductive and survival structures, we were able to re-isolate the $c m k 3$ mutant from the chlorotic spots when the emerging hyphae were transferred to PDA supplemented with hygromycin.

\section{Complementation of $c z k 3$.}

To determine whether the mutant phenotype could be complemented by introduction of the wild-type $C Z K 3$ gene, we transformed disrupted mutant protoplasts with the complementation vector pKB501 containing an 11-kb fragment comprised of upstream and downstream flanking sequences as well as the entire open reading frame. Complemented transformants were selected based on bialophos resistance and cercosporin production on $0.2 \times$ PDA. Southern analysis of a complemented transformant (M1228CP23) revealed both the wild-type gene (3 kb) and the disrupted copy (4.4 kb) (Fig. 6). This complemented strain grew at the wild-type rate and produced $>80 \%$ of the wild-type level of cercosporin after 10 days. The ability to produce conidiophores and conidia on V8 juice agar was fully restored by complementation (Table 1). Furthermore, the complemented strain elicited necrotic lesions identical to those incited by the wild type and could be re-isolated from the lesions onto PDA supplemented with hygromycin. The data indicated that disrupted $c z k 3$ is responsible for the multiple phenotypes observed in mutant M1228-21.

Table 1. Radial growth, conidiation, and cercosporin production by Cercospora zeae-maydis wild type (CZK3), disrupted mutant (czk3), and complemented strain $(C Z K 3-1)$

\begin{tabular}{|c|c|c|c|}
\hline Trait & CZK3 & czk3 & CZK3-1 \\
\hline${\text { Radial growth }(\mathrm{mm})^{\mathrm{a}}}^{\mathrm{a}}$ & $15.0 \pm 1.0(100)^{b}$ & $29.3 \pm 1.5(196)$ & $15.3 \pm 0.6(102)$ \\
\hline Conidia $\left(\times 10^{3} / \mathrm{cm}^{2}\right)^{\mathrm{c}}$ & $39.8 \pm 4.5(100)$ & $0(0)$ & $40.7 \pm 6.6(102)$ \\
\hline Cercosporin $(\mathrm{ng} / \mathrm{ml})^{\mathrm{d}}$ & $32.5 \pm 10.3(100)$ & $0(0)$ & $26.8 \pm 11.7(82.5)$ \\
\hline
\end{tabular}

\footnotetext{
${ }^{\text {a }}$ Colony diameter after 10 days growth on potato dextrose agar (PDA) at $25^{\circ} \mathrm{C}$.

${ }^{\mathrm{b}}$ Values are means \pm standard deviation of three replicates, and numbers in parentheses are the percent of wild-type values.

${ }^{\mathrm{c}}$ Conidia production after 3 days in 14-h light and 10 -h dark cycle on V8 juice agar at $25^{\circ} \mathrm{C}$.

${ }^{\mathrm{d}}$ Cercosporin concentration after 10 days growth on $0.2 \times \mathrm{PDA}$ at $25^{\circ} \mathrm{C}$.
} 


\section{DISCUSSION}

Although other components of signal transduction pathways have been shown to influence the linkage between secondary metabolism and fungal development or between development and pathogenesis, CZK3 is the first MAPKKK identified in filamentous fungi that influences all three processes-secondary metabolism, conidiation, and pathogenesis. Targeted disruption of $C Z K 3$ abolished cercosporin production, significantly suppressed expression of $P M O$ and $L D S$ and decreased expression of $D P O$ to a level approximately half of that observed in the wild type (Fig. 4). Those were among genes proposed to be involved in cercosporin biosynthesis (Shim and Dunkle 2002) and may be considered tenable candidates because cytochrome P450 monooxygenases and oxidases are directly involved in structural modifications of fungal secondary metabolites (Alexander et al. 1998; Brown et al. 1996b; Huang et al. 1995; Martasek et al. 1994). In addition, enzymes in lipid metabolism are involved in the synthesis and modification of precursors in secondary metabolic pathways (Ahn and Walton 1998; Brobst and Townsend 1994; Brown et al. 1996a and b).

Disruption of $C Z K 3$ increased vegetative growth but blocked conidiation. The potential connection between cercosporin synthesis, growth rate, and conidiation also was suggested by previous work on $C$. kikuchii. UV-induced mutants of $C$. kikuchii blocked in cercosporin production grew more rapidly on agar media and produced $<1 \%$ of the amount of conidia as wild type (Upchurch et al. 1991). Results of that study and earlier work by Jenns and associates (1989) indicated that cercosporin production is correlated with reduced growth on agar media. On the other hand, cercosporin-deficient, CFP disruptants, which were (presumably) deficient in sporulation, since mycelium was used as inoculum in pathogenicity assays, were not diminished in growth or viability (Callahan et al. 1999) and no evidence was presented to show that growth of the mutants was enhanced over wild type. We have obtained disruption mutants that are deficient in cercosporin production but conidiate normally, suggesting that the two processes are not consistently and necessarily linked (W.-B. Shim and L. D. Dunkle, unpublished data).

The relationship between fungal secondary metabolism and sporulation has been studied in other fungi. For example, $A$. nidulans contains a cluster of genes that encode enzymes required to synthesize sterigmatocystin, a precursor of aflatoxin (Brown et al. 1996b). One sterigmatocystin cluster (STC) gene, $A F L R$, functions as a pathway-specific transcriptional regulator for activation of other genes in the sterigmatocystin pathway (Woloshuk et al. 1994). Hicks and associates (1997) showed that FLBA, which encodes an RGS domain protein, and $F A D A$, which encodes a subunit of a heterotrimeric $\mathrm{G}$ protein, regulate sterigmatocystin production and asexual sporulation. Overexpression of FLBA or dominant interfering FADA mutations resulted in precocious STC gene expression and accumulation of sterigmatocystin and irregular sporulation (Hicks et al. 1997). The ability of FLBA to activate STC gene expression is dependent upon $F L U G$, another early acting developmental regulator, and $A F L R$, the STC gene-specific transcription factor (Hicks et al. 1997). Shimizu and Keller (2001) showed that a cAMP-dependent protein kinase A mediates the signaling pathways regulating asexual development and sterigmatocystin biosynthesis. Similar relationships have been documented for the maize ear rot fungus, $F$. verticillioides, which produces fumonisins in maize kernels. Shim and Woloshuk (2001) generated a mutation that disrupted the FCC1 gene encoding a C-type cyclin, which resulted in altered fumonisin B1 biosynthesis. When the mutant was grown on maize kernels or on defined minimal medium at $\mathrm{pH} 6$, conidiation was reduced, and expression of FUM5, the polyketide synthase gene in- volved in fumonisin B1 biosynthesis, was abolished. The results suggested that $F C C 1$ plays an important role in signal transduction regulating fumonisin biosynthesis and conidiation in $F$. verticillioides.

Our data indicate that Czk3 is critical for disease development and expression of wild-type virulence (Fig. 5). The $c z k 3$ disruption mutant could penetrate leaves and initiate disease but was incapable of developing fully expanded lesions (Fig. 5A), whereas the wild type, although initially slower to elicit chlorosis, ultimately caused typical gray leaf spot lesions. Wild-type virulence was restored by complementation of the disrupted mutant with the $C Z K 3$ gene. These observations suggest that cercosporin is required for full expression of typical gray leaf spot symptoms but not for initiation of infection. However, further study is needed to support a conclusion that cercosporin is a virulence factor in C. zeae-maydis, because mutation in $C Z K 3$ caused pleiotropic phenotypes. Characterization of genes that are directly involved in cercosporin biosynthesis, such as $P M O$ and $L D S$, is in progress and will provide results to test this hypothesis directly.

MAP kinase pathways are closely associated with fungal development and pathogenesis (Dickman and Yarden 1999; Xu 2000). Signal transduction pathways regulating host recognition, appressorium formation, and invasive growth in $M$. grisea have been identified (Dean 1996). Two MAP kinases involved in pathogenicity of the rice blast fungus are $P M K 1$ (pathogenicity MAP kinase) and MPS1 (MAP kinase for penetration and sporulation) (Xu and Hamer 1996; Xu et al. 1998). Although $P M K 1$ is not essential for vegetative growth or sexual and asexual reproduction in culture, pmkl mutants are nonpathogenic and fail to develop lesions on susceptible rice cultivars (Xu and Hamer 1996). The M. grisea mpsl mutants are nonpathogenic on rice leaves but, unlike pmkl mutants, they can infect rice leaves through wounds. MPS1 was shown to be essential for penetration by appressoria, and mps 1 mutants were drastically reduced in conidiation and development of aerial hyphae (Xu et al. 1998).

In $U$. maydis, a dimorphic basidiomycete that causes corn smut, the haploid yeast form is nonpathogenic, and the dikaryon formed by hyphal fusion between two compatible haploid strains is pathogenic. Consequently, genes that regulate conjugation or filamentous growth necessarily affect the infection process, and mutations in those genes abolish pathogenicity. The cAMP signaling and MAP kinase pathways have been implicated in regulating pheromone response, cell fusion, filamentous growth, and pathogenesis (Kronstad et al. 1998). Disruption of the adenylate cyclase gene (uacl) results in constitutive filamentous growth (Gold et al. 1994). A systematic search for suppressor mutations of the $u a c l$ mutant resulted in the identification of $u b c 4$, a MAPKKK, $u b c 5$, a MAPKK (Andrews et al. 2000), and $u b c 3$, the MAP kinase (Mayorga and Gold 1998, 1999). Thus, $u b c 4$, $u b c 5$, and $u b c 3$ likely comprise a MAP cascade that interacts with cAMP signaling to control mating and processes associated with pathogenesis. The $u b c 5$ homolog $f u z 7$ was shown previously to be required for pheromone-responsive morphogenesis (Banuette and Herskowitz 1994).

Sequence analysis of full-length $C Z K 3$ showed that the Czk3 protein is highly similar to Wis4 of fission yeast $S$. pombe. Wis4 is a MAPKKK in the Spc1 MAP kinase pathway, which is involved in various stress responses (Samejima et al. 1997, 1998). Integrity of the Wis1 (MAPKK) and Spc1 (MAPK) pathway is required for survival of $S$. pombe under several stress conditions, e.g., osmotic, heat, or oxidative stresses (Banuette 1998; Degols et al. 1996; Kato et al. 1996) and is involved in cell cycle progression and control of sexual development in $S$. pombe (Kato et al. 1996; Millar et al. 1995; Warbrick and Fantes 1991). Although most closely related to Hog1, a MAP 
kinase specifically responsive to osmotic stress in $S$. cerevisiae, Spc1, also known as Sty1 (Banuette 1998; Millar et al. 1995), is activated by multiple forms of stress, including exposure to high osmolarity or growth in suboptimal nutrient conditions. Unlike the defined MAP kinase pathways of $S$. cerevisiae, which perform distinct functions, the Spc1 MAP kinase pathway performs multiple functions in $S$. pombe. Significantly, the Spc1 pathway uses two MAPKKKs, Win1 and Wis4, to effectively respond to different stresses (Samejima et al. 1997, 1998). Win1 is responsible for the high osmolarity response, whereas Wis4 is involved in recognition of heat, oxidative, and nutrient stresses.

Production of cercosporin by C. zeae-maydis is maximal in nutrient-poor media, such as dilute PDB, and is minimal or entirely absent in rich media (W.-B. Shim and L. D. Dunkle, unpublished data). The fact that Czk3 is highly similar to Wis4 suggests that nutrient stress activates the cercosporin biosynthetic pathway. Addition of ammonium salts to $0.2 \times$ PDB effectively decreases expression of $P M O, L D S$, and $D P O$ and suppresses cercosporin production (Shim and Dunkle 2002), suggesting that cercosporin biosynthesis may be triggered by nitrogen status. Consistent with nitrogen-regulated genes in other filamentous fungi (Chang et al. 1996; Hass and Marzluf 1995; Tudzynski et al. 1999), identification of a GATA-binding factor in the promoter region of $C Z K 3$ supports this hypothesis.

In addition to the lack of cercosporin production, one of the most obvious phenotypes observed in the $c m k 3$ mutant is the absence of asexual reproductive structures. On V-8 juice agar (Fig. 2) and on inoculated maize leaves (Fig. 5B), the $c z k 3 \mathrm{mu}-$ tant was blocked in production of stromata and conidiophores. These reproductive structures are essential for producing primary and secondary inoculum for gray leaf spot. Without proper function of $C Z K 3$, the progression of gray leaf spot would cease after limited development within single infection sites. Thus, mutation in $C Z K 3$ not only inhibits fungal reproduction but, as a result, also disrupts the disease cycle of gray leaf spot. In this regard, $C Z K 3$ may be considered a pathogenicity gene as well as a virulence gene.

\section{MATERIALS AND METHODS}

Fungal strain, media, and cercosporin assay.

A monoconidial isolate of C. zeae-maydis strain SCOH1-5 was recovered from a gray leaf spot lesion and maintained as described previously (Payne and Waldron 1982; Shim and Dunkle 2002). The fungus was grown on $0.2 \times$ PDA (Difco, Detroit, MI, U.S.A.) to evaluate production of the reddish-pigmented cercosporin and on V-8 juice agar to obtain conidia (Wang et al. 1998). Cercosporin was quantified by spectrophotometric analysis of alkali extracts of mycelia-agar plugs, as described previously (Jenns et al. 1989; Shim and Dunkle 2002). To quantify conidiation, agar cylinders were excised with a cork borer $(1 \mathrm{~cm}$ diameter) from a 3-day-old V-8 juice agar culture (Lapaire and Dunkle 2003), were placed in a test tube with $1 \mathrm{ml}$ of sterile water, and were vortexed to dislodge the conidia, which were counted with a hemacytometer.

\section{Nucleic acid manipulation.}

Bacterial plasmids were isolated with the Wizard DNA purification system (Promega, Madison, WI, U.S.A.). Genomic DNA of C. zeae-maydis was isolated from mycelium grown in PDB (Difco) or complete medium (CM) broth, as described previously (Shim and Dunkle 2002; Woloshuk et al. 1995). Total RNA was isolated with Trizol reagent (Gibco BRL, Grand Island, NY, U.S.A.), according to the manufacturer's suggested protocol. Southern and Northern analyses were performed as described previously (Sambrook et al. 1989; Shim and Dunkle 2002). DNA probes were ${ }^{32} \mathrm{P}$-labeled with a Prime-It II random primer labeling kit (Stratagene, La Jolla, CA, U.S.A.).

\section{Construction of $C$. zeae-maydis genomic library and bacterial filter array.}

Mycelia of C. zeae-maydis grown in CM broth were harvested by filtration through Miracloth (Calbiochem, La Jolla, CA, U.S.A.), were frozen in liquid nitrogen, and were ground to a fine powder. Genomic DNA was extracted and dissolved in TE buffer $(10 \mathrm{mM}$ Tris- $\mathrm{HCl}$ and $1 \mathrm{mM}$ EDTA, $\mathrm{pH} 8)$ at $4^{\circ} \mathrm{C}$ overnight without agitation. A C. zeae-maydis genomic library was constructed with the SuperCos1 cosmid vector kit (Stratagene). A total of 3,072 clones were selected, and six replicates of each clone were applied to a bacterial filter array $(22 \times 2 \mathrm{~cm})$ at the Agricultural Genome Center, Purdue University. After incubation on the filters, the resulting colonies were lysed, and their DNAs cross linked to the filter with UV irradiation.

\section{Isolation and sequencing of the cosmid containing $C Z K 3$.}

A ${ }^{32}$ P-labeled cDNA insert (380 bp) obtained from clone P02K17 was used as a probe to identify a cosmid clone (bcM3K) containing the $C Z K 3$ gene. To obtain the nucleotide sequence of $C Z K 3$, a 3-kb SphI fragment from bcM3K, which hybridized to the ${ }^{32} \mathrm{P}$-labeled cDNA insert, was subcloned into pGEM-3Zf(+) vector (Promega). The resulting plasmid pKB307 was submitted to the DNA Sequencing and Synthesis Facility (Iowa State University, Ames, IA, U.S.A.), for primer walking. Concurrently, we used the EZ::TN <TET-1> insertion kit (Epicentre, Madison, WI, U.S.A.) to generate random transposon insertions in bcM3K and used the forward and reverse sequencing primers provided in the kit to perform sequencing. DNA sequencing and subsequent contig assembly were performed at the Agricultural Genome Center, Purdue University. Sequences were analyzed via the BLAST algorithm (Altschul et al. 1997), and multiple amino acid sequences were aligned via CLUSTAL W (Thompson et al. 1994).

\section{Fungal transformation.}

C. zeae-maydis conidia $\left(10^{7}\right)$ were inoculated into $100 \mathrm{ml}$ of $0.2 \times \mathrm{PDB}$ and incubated for $72 \mathrm{~h}$ at $25^{\circ} \mathrm{C}$ on a rotary shaker at $150 \mathrm{rpm}$. Protoplasts were prepared from the vegetative hyphae as described by Shim and Woloshuk (2001). Disruption vector pKB102 was constructed by inserting the $H P H$ gene into the EcoRV site of the pP02K17 cDNA fragment (Fig. 1A). Protoplasts were transformed as described previously (Callahan et al. 1999; Shim and Woloshuk 2001), were regenerated in CZM regeneration broth (CM broth, except $1 \mathrm{M}$ sucrose was substituted for $56 \mathrm{mM}$ glucose) overnight at $25^{\circ} \mathrm{C}$ on a rotary shaker at $150 \mathrm{rpm}$, and were plated in CZM regeneration agar the following day. After 7 days of incubation at $25^{\circ} \mathrm{C}$, top agar (PDA containing $150 \mu \mathrm{g}$ of hygromycin $\mathrm{B}$ per $\mathrm{ml}$ ) was applied to the plates. After 7 days of incubation at $25^{\circ} \mathrm{C}, 63$ hygromycin-resistant transformants were selected, were transferred to $0.2 \times$ PDA for cercosporin production, and were subjected to Southern analysis of genomic DNA digested with SphI and probed with the 380-bp insert from clone pP02K17. Transformants with ectopic integration of the disruption vector produced wildtype levels of cercosporin. Southern analysis of one hygromycin-resistant transformant (M1228-21), which did not produce cercosporin, indicated a disrupted $C Z K 3$ gene.

Complementation of $c z k 3$ in disrupted mutant M1228-21 was performed with vector pKB501 (Fig. 1B). This complementation vector was constructed by subcloning the $11-\mathrm{kb}$ Bam HI fragment from bcM3K into vector pBARGPE1, which contains the phosphinothricin acetyltransferase $(B A R)$ gene conferring resistance to bialophos (commercial products Ignite 
or Basta) ligated to the A. nidulans TRPC promoter and terminator (Pall and Brunelli 1993). Transformants were regenerated in $\mathrm{CZM}$ regeneration broth at $25^{\circ} \mathrm{C}$ for $18 \mathrm{~h}$ on a rotary shaker at $150 \mathrm{rpm}$. Regenerated protoplasts were incubated in CZM regeneration agar for 7 days before top agar (PDA with $50 \mu \mathrm{g}$ of bialophos per ml) was applied. Colonies were selected as putative complements based on bialophos resistance and cercosporin production.

\section{RT-PCR.}

RT-PCR amplifications were performed in a 50- $\mu$ l reaction mixture with the Access RT-PCR system (Promega) in a DNA thermal cycler (Model 480, Perkin Elmer Cetus, Torrance, CA, U.S.A.). The primers for RT-PCR reactions were as follows: for FAS, pFAS3 (5'-AGCAAGTCCGAGCTTGCC-3') and pFAS5 (5'-CAGTCTCCTTGGACGACT-3'); for LDS, pLDSF1 (5'TGCCTGACAGCTTCTTGC-3') and pLDSR1 (5'-GTTGCAC ACCTCCATGTCC-3'); for DHO, pDHOF1 (5'-CGAGACG CTGTTGGAGC-3') and pDHOR1 (5'-GCCATTGTTGAAG TTGGAGC-3'); for STC, pSTCF1 (5'-CGAGCTCAGTTTTC TGGACG-3') and pSTCR1 (5'-CTGGAAAGTCAGATGAGG ACG-3'); for PDX1, Sor1fs (5'-ACAAAGGGTGAAGCAG GAAT-3') and Sor1ra (5'-GGCAGCTTGTCGCAGTTGAT-3') (Shim and Dunkle 2002). The reactions containing $15 \mu \mathrm{g}$ of total RNA from wild type and mutant mycelia grown for 7 days in $0.2 \times$ PDB were carried out according to the manufacturer's suggested protocol, except that the annealing temperature was $58^{\circ} \mathrm{C}$.

\section{Pathogenicity assay.}

To obtain inoculum for plant infection, wild-type (SCOH15) and $c z k 3$ mutant (M1228-21) isolates were grown in $0.2 \times$ PDB for 7 days at $25^{\circ} \mathrm{C}$ on a rotary shaker at $150 \mathrm{rpm}$, and cultures were harvested by filtration through Miracloth. mycelial mats $(2 \times 2 \mathrm{~mm})$ were placed on the abaxial surface of Pioneer brand 3394 maize leaves (L8 stage). The plants were incubated in a dew chamber in the greenhouse at $>98 \%$ relative humidity, and symptom expression was observed daily (Fig. 5A). As an alternative method, mycelium was washed with distilled water and was homogenized in a sterile stainless steel blender, and the mycelial fragments were sprayed onto maize leaves. The inoculated plants were incubated in a growth chamber maintained at $>98 \%$ relative humidity and $25^{\circ} \mathrm{C}$ under a 14-h light and 10-h dark cycle.

To assess fungal growth in planta, leaf samples bearing lesions and surrounding area were cut into $1 \times 2$-cm sections. These sections were cleared in ethanol/acetic acid $(1: 1$ $\mathrm{vol} / \mathrm{vol}$ ) at $55^{\circ} \mathrm{C}$ and then incubated in $0.05 \%$ (wt/vol) cotton blue in lactophenol to stain the fungal mycelium (Fig. 5B). The stained sections were mounted on glass slides with lactophenol and viewed with a Nikon Labophot light microscope (Kanagawa, Japan), and fungal growth was recorded with a digital camera.

\section{ACKOWLEDGMENTS}

Research reported is a cooperative investigation of the United States Department of Agriculture Agricultural Research Service and the Purdue University Agricultural Experiment Station. Published as paper no. 17069, Purdue University Agricultural Experiment Station. The authors thank M. McClenning and C. Lapaire for excellent technical assistance.

\section{LITERATURE CITED}

Adams, T. H., and Yu, J. H. 1998. Coordinate control of secondary metabolite production and asexual sporulation in Aspergillus nidulans. Curr. Opin. Microbiol. 6:674-677.
Ahn, J.-H., and Walton, J. D. 1997. A fatty acid synthase gene in Cochliobolus carbonum required for production of HC-Toxin, cyclo (D-prolyl-L-alanyl-D-alanyl-L-2-amino-9,10-epoxi-8-oxodecanoyl). Mol. PlantMicrobe Interact. 10:207-214.

Alexander, N. J., Hohn, T. M., and McCormick, S. P. 1998. The TRI11 gene of Fusarium sporotrichioides encodes a cytochrome P-450 monooxygenase required for $\mathrm{C}-15$ hydroxylation in trichothecene biosynthesis. Appl. Environ. Microbiol. 64:221-225.

Altschul, S. F., Madden, T. L., Schaffer, A. A., Zhang, J. H., Zhang, Z., Miller, W., and Lipman, D. J. 1997. Gapped BLAST and PSI-BLAST: A new generation of protein database search program. Nucleic Acids Res. 25:3389-3402.

Andrews, D. L., Egan, J. D., Mayorga, M. E., and Gold, S. E. 2000. The Ustilago maydis ubc4 and $u b c 5$ genes encode members of a MAP kinase cascade required for filamentous growth. Mol. Plant-Microbe Interact. 13:781-786.

Assante, G., Locci, R., Camarda, L., Merlini, L., and Nasini, G. 1977. Screening of the genus Cercospora for secondary metabolites. Phytochemistry 16:243-247.

Banuette, F. 1998. Signaling in yeast: An information cascade with links to the filamentous fungi. Microbiol. Mol. Biol. Rev. 62:249-271.

Banuette, F., and Herskowitz, I. 1994. Identification of Fuz7, a Ustilago maydis MAPKK homologue required for a locus-dependent and -independent steps in the fungal life cycle. Genes Dev. 8:1367-1378.

Brobst, S. W., and Townsend, C. A. 1994. The potential role of fatty acid initiation in the biosynthesis of the fungal aromatic polyketide aflatoxin B1. Can. J. Chem. 72:200-207.

Brown, D. W., Adams, T. H., and Keller, N. P. 1996a. Aspergillus has distinct fatty acid synthases for primary and secondary metabolism. Proc. Natl. Acad. Sci. U.S.A. 93:14873-14877.

Brown, D. W., Yu, J. H., Kelkar, H. S., Fernandes, M., Nesbitt, T. C., Keller, N. P., Adams, T. H., and Leonard, T. J. 1996b. Twenty-five coregulated transcripts define a sterigmatocystin gene cluster in Aspergillus nidulans. Proc. Natl. Acad. Sci. U.S.A. 93:1418-1422.

Callahan, T. M., Rose, M. S., Meade, M. J., Ehrenshaft, M., and Upchurch, R. G. 1999. CFP, the putative cercosporin transporter of Cercospora kikuchii, is required for wild type cercosporin production, resistance, and virulence on soybean. Mol. Plant-Microbe Interact. 12:901-910.

Chang, P. K., Ehrlich, K. C., Linz, J. E., Bhatnagar, D., Cleveland, T. E., and Bennet, J. W. 1996. Characterization of the Aspergillus parasiticus niaD and niiA gene cluster. Curr. Genet. 30:68-75.

Daub, M. E. 1982. Cercosporin, a photosensitizing toxin from Cercospora species. Phytopathology 72:370-374.

Daub, M. E., and Ehrenshaft, M. 2000. The photoactivated Cercospora toxin cercosporin: Contribution to plant disease and fundamental biology. Annu. Rev. Phytopathol. 38:461-490.

Dean, R. A. 1996. Signal pathways and appressorium morphogenesis. Annu. Rev. Phytopathol. 35:211-234.

Degols, G., Shiozaki, K., and Russell, P. 1996. Activation and regulation of the Spc1 stress-activated protein kinase in Schizosaccharomyces pombe. Mol. Cell. Biol. 16:2870-2877.

Dickman, M. B., and Yarden, O. 1999. Serine/threonine protein kinases and phosphatases in filamentous fungi. Fungal Genet. Biol. 26:99-117.

Ehrenshaft, M., Bilski, P., Li, M. Y., Chignell, C. F., and Daub, M. E. 1999. A highly conserved sequence is a novel gene involved in de novo vitamin B6 biosynthesis. Proc. Natl. Acad. Sci. U.S.A. 96:9374-9378.

Ehrenshaft, M., Jenns, A. E., Chung, K. R., and Daub, M. E. 1998. SOR1, a gene required for photosensitizer and singlet oxygen resistance in Cercospora fungi, is highly conserved in divergent organisms. Mol. Cell 1:603-609.

Fajola, A. O. 1978. Cercosporin, a phytotoxin from Cercospora spp. Physiol. Plant Pathol. 13:157-164.

Gold, S., Brogdon, S. M., Mayorga, M. E., and Kronstad, J. W. 1994. cAMP regulates morphogenesis in the fungal pathogen Ustilago maydis. Genes Dev. 8:2805-2816.

Gwinn, K. D., Stelzig, D. A., and Brooks, J. L. 1987. Effects of corn plant-age and cultivar on resistance to Cercospora zeae-maydis and sensitivity to cercosporin. Plant Dis. 71:603-606.

Hass, H., and Marzluf, G. A. 1995. NRE, the major nitrogen regulatory protein of Penicillium chrysogenum, binds specifically to elements in the intergenic promoter regions of nitrate assimilation and penicillin biosynthetic gene clusters. Curr. Genet. 28:177-183.

Hicks, J. K., Yu, J. H., Keller, N. P., and Adams, T. H. 1997. Aspergillus sporulation and mycotoxin production both require inactivation of the FadA G alpha protein-dependent signaling pathway. EMBO (Eur. Mol. Biol. Organ.) J. 16:4916-4923.

Huang, K. X., Fujii, I., Ebizuka, Y., Gomi, K., and Sankawa, U. 1995. Molecular cloning and heterologous expression of the gene encoding 
dihydrogeodin oxidase, a multicopper blue enzyme from Aspergillus terreus. J. Biol. Chem. 270:21495-21502.

Jenns, A. E., Daub, M. E., and Upchurch, R. G. 1989. Regulation of cercosporin accumulation in culture by medium and temperature manipulation. Phytopathology 79:213-219.

Kato, T., Okazaki, K., Murakami, H., Stettler, S., Fantes, P. A., and Okayama, H. 1996. Stress signal, mediated by a Hog1-like MAP kinase, controls sexual development in fission yeast. FEBS (Fed. Eur. Biochem. Soc.) Lett. 378:207-212.

Kronstad, J., Maria, A. D., Funnell, D., Laidlaw, R. D., Lee, N., Mario, M. D., and Ramesh, M. 1998. Signaling via cAMP in fungi: Interconnections with mitogen activated protein kinase pathways. Arch. Microbiol. 170:395-404

Kuyama, S., and Tamura, T. 1957. Cercosporin. A pigment of Cercosporina kikuchii Matsumoto et Tomoyasu. I. Cultivation of fungus, isolation and purification of pigment. J. Am. Chem. Soc. 79:57255726.

Lapaire, C. L., and Dunkle, L. D. 2003. Microcycle conidiation in Cercospora zeae-maydis. Phytopathology 93:193-199.

Lynch, F. J., and Geoghegan, M. J. 1979. Regulation of growth and cercosporin photoinduction in Cercospora beticola. Trans. Br. Mycol. Soc. 73:311-327.

Martasek, P., Camadro, J. M., Delfaularue, M. H., Dumas, J. B., Montagne, J. J., Deverneuil, H., Labbe, P., and Grandchamp, B. 1994 Molecular cloning, sequencing, and functional expression of a cDNAencoding human coproporphyrinogen oxidase. Proc. Natl. Acad. Sci. U.S.A. 91:3024-3028.

Mayorga, M. E., and Gold, S. E. 1998. Characterization and molecular genetic complementation of mutants affecting dimorphism in the fungus Ustilago maydis. Fungal Genet. Biol. 24:364-376.

Mayorga, M. E., and Gold, S. E. 1999. A MAP kinase encoded by the $u b c 3$ gene of Ustilago maydis is required for filamentous growth and full virulence. Mol. Microbiol. 34:485-497.

Millar, J. B. A., Buck, V., and Wilkinson, M. G. 1995. Pyp1 and Pyp2 PTPases dephosphorylate an osmosensing MAP kinase controlling cell size at division in fission yeast. Genes Dev. 9:2117-2130.

Muller, P., Aichinger, C., Feldbrugge, M., and Kahmann, R. 1999. The MAP kinase Kpp2 regulates mating and pathogenic development in Ustilago maydis. Mol. Microbiol. 34:1007-1017.

Pall, M. L., and Brunelli, J. P. 1993. A series of six compact fungal transformation vectors containing polylinkers with multiple unique restriction sites. Fungal Genet. Newsl. 40:59-62.

Posas, F., and Saito, H. 1998. Activation of the yeast SSK2 MAP kinase kinase kinase by the SSK1 two-component response regulator. EMBO (Eur. Mol. Biol. Organ.) J. 17:1385-1394.

Payne, G. A., and Waldron, J. K. 1983. Overwintering and spore release of Cercospora zeae-maydis in corn debris in North Carolina. Plant Dis. 67:87-89.

Sambrook, J., Fritsch, E. F., and Maniatis, T. 1989. Molecular cloning: A Laboratory Manual. 2nd ed. Cold Spring Harbor Laboratory, Cold Spring Harbor, NY, U.S.A

Samejima, I., Mackie, S., and Fantes, P. A. 1997. Multiple modes of activation of the stress-responsive MAP kinase pathway in fission yeast. EMBO (Eur. Mol. Biol. Organ.) J. 16:6162-6170.

Samejima, I., Mackie, S., Warbrick, E., Weisman, R., and Fantes, P. A. 1998. The fission yeast mitotic regulator $w i n I^{+}$encodes a MAP kinase kinase kinase that phosphorylates and activates Wis1 MAP kinase kinase in response to high osmolarity. Mol. Biol. Cell 9:2325-2335.

Shim, W.-B., and Dunkle, L. D. 2002. Identification of genes expressed during cercosporin biosynthesis in Cercospora zeae-maydis. Physiol. Mol. Plant Pathol. 61:237-248.

Shim, W.-B., and Woloshuk, C. P. 2001. Regulation of fumonisin B1 bio- synthesis and conidiation in Fusarium verticillioides by a cyclin-like (C-type) gene, FCC1. Appl. Environ. Microbiol. 67:1607-1612.

Shimizu, K., and Keller, N. P. 2001. Genetic involvement of a cAMP-dependent protein kinase in a $\mathrm{G}$ protein signaling pathway regulating morphological and chemical transitions in Aspergillus nidulans. Genetics 157:591-600.

Tenjo, F., Xu, J. R., and Hamer, J. E. 1999. Expression and cellular distribution of the Magnaporthe grisea MAP kinase PMK1. Fungal Genet. Newsl. 46:118.

Thompson, J. D., Higgins, D. G., and Gibson, T. J. 1994. CLUSTAL W: Improving the sensitivity of progressive multiple sequence alignment through sequence weighting, position-specific gap penalties and weight matrix choice. Nucleic Acids Res. 22:4673-4680.

Tudzynski, B., Homann, V., Feng, B., and Marzluf, G. A. 1999. Isolation, characterization and disruption of the areA nitrogen regulatory gene of Gibberella fujikuroi. Mol. Gen. Genet. 261:106-114.

Upchurch, R. G., Walker, D. C., Rollins, J. A., Ehrenshaft, M., and Daub, M. E. 1991. Mutants of Cercospora kikuchii altered in cercosporin synthesis and pathogenicity. Appl. Environ. Microbiol. 57:2940-2945.

Velicheti, R. K., and Sinclair, J. B. 1994. Production of cercosporin and colonization of soybean seed coats by Cercospora kikuchii. Plant Dis. 78:342-346.

Venkataramani, K. 1967. Isolation of cercosporin from Cercospora personata. Phytopathol. Z. 58:379-382.

Wang, J., Levy, M., and Dunkle, L. D. 1998. Sibling species of Cercospora associated with gray leaf spot of maize. Phytopathology $88: 1269-1275$

Warbrick, E., and Fantes, P. A. 1991. The wis1 protein is a dosage-dependent regulator of mitosis in Schizosaccharomyces pombe. EMBO (Eur. Mol. Biol. Organ.) J. 10:4291-4299.

Woloshuk, C. P., Foutz, K. R., Brewer, J. F., Bhatnagar, D., Cleveland, T. E., and Payne, G. A. 1994. Molecular characterization of aflR, a regulatory locus for aflatoxin biosynthesis. Appl. Environ. Microbiol. 60:2408-2414

Woloshuk, C. P., Yousibova, G. L., Rollins, J. A., Bhatnagar, D., and Payne, G. A. 1995. Molecular characterization of the Afl-1 locus in Aspergillus flavus. Appl. Environ. Microbiol. 61:3019-3023.

Wolpert, T. J., Dunkle, L. D., and Ciuffetti, L. M. 2002. Host-selective toxins and avirulence determinants: What's in a name? Annu. Rev. Phytopathol. 40:252-285.

Xu. J. R. 2000. MAP kinases in fungal pathogens. Fungal Genet. Biol. 31:137-152.

Xu, J. R., and Hamer, J. E. 1996. MAP kinase and cAMP signaling regulates infection structure formation and pathogenic growth in the rice blast fungus Magnaporthe grisea. Genes Dev. 10:2696-2706.

Xu, J. R., Staiger, C. J., and Hamer, J. E. 1998. Inactivation of the mitogen-activated protein kinase MPS1 from the rice blast fungus prevents penetration of host cells but allows activation of plant defense responses. Proc. Natl. Acad. Sci. U.S.A. 95:12713-12718.

Yoder, O. C. 1980. Toxins in pathogenesis. Annu. Rev. Phytopathol. 18:103-129.

Yu, J. H., Wieser, J., and Adams, T. H. 1996. The Aspergillus FlbA RGS domain protein antagonizes $\mathrm{G}$ protein signaling to block proliferation and allow development. EMBO J. 19:5184-5190.

\section{AUTHOR-RECOMMENDED INTERNET RESOURCES}

Center for Genome Research, Whitehead Institute index of annotation/ fungi: www-genome.wi.mit.edu/annotation/fungi/

Transcription Regulation Element Search tool for comparative promoter analysis: bioportal.bic.nus.edu.sg/tres/ 